\title{
ПРИМЕНЕНИЕ ДЫРОЧНОЙ ГРУППОВОЙ МОДЕЛИ К СИСТЕМАМ С НЕНАСЫЩЕННЫМИ УГЛЕВОДОРОДАМИ
}

\author{
(Представиљ О. Киррет)
}

Для описания термодинамических характеристик систем в настоящее время широко используются групповые модели раствора. Обращение к ним продиктовано возможностью оценки свойств большого числа систем по ограниченному объему исходных данных. Наряду с применением весьма популярных эмпирических моделей, УНИФАК ['] и АСОГ [ $\left.{ }^{2}\right]$, систематически исследуются теоретически более последовательные квазихимические модели. Их развитие открывает возможность для широкого охвата термодинамических свойств жидкостей и смесей.

Одна из таких моделей, дырочная квазихимическая модель [3], позволила описать многие термодинамические свойства большого числа индивидуальных гомологов алканов и алканолов и содержащих их смесей $\left[{ }^{3-5}\right]$.

В настоящей работе приведены результаты применения этой модели к системам с ненасыщенными углеводородами. Целью ее была проверка возможности описания одними и теми же параметрами парожидкостного равновесия и энтальпий смешения в системах, образованных 1-алкенами и 1-алкинами с $\boldsymbol{H}$-алканами.

В основе группового варианта указанной модели лежат общее для групповых моделей предположение о независимости характеристик групп от принадлежности к конкретной молекуле. Модель допускает наличие вакансий (дырок) в квазирешетке и учитывает размер и форму молекул, а также ориентационные эффекты в квазихимическом приближении. Молекула сорта $i$ занимает $r_{i}$ мест в решетке, каждой дырке отводится одно место $\left(r_{0}=1\right)$. Дырки рассматриваются как сорт частиц смеси. «Химический потенциал» компонента $i$ в такой формально $(K+1)$-компонентной смеси выражается через

$$
\tilde{\mu}_{i}=\left(\partial F / \partial N_{i}\right)_{T, N_{j \neq i}}, \quad i=0,1, \ldots, k,
$$

где $F-$ энергия Гельмгольца, $N_{i}$ - число молекул сорта $i$.

Связь с химическим потенциалом $\mu_{i}=\left(\partial F / \partial N_{i}\right)_{T, v, N_{j \neq i}}$, где $V$ - объем, определяется соотношением

$$
\mu_{i}=\tilde{\mu}_{i}-\tilde{r}_{i} \tilde{\mu}_{0}, \quad i=1, \ldots, k
$$

Давление $P$ связано с «химическим потенциалом» дырок через

$$
\tilde{\mu}_{0}=-P v^{*},
$$

где $v^{*}$ - объем, отвечающий одному месту в квазпрешетке $\left(v^{*}=14,244\right.$ $\mathrm{cm}^{3} /$ моль).

Считая стандартными чистые жидкости в равновесии с паром при температуре раствора и приписывая им верхний индекс $i$, получают выражение для коэффициента активности компонента $i\left(\gamma_{i}\right)$ 


$$
\ln x_{i} \gamma_{i}=\ln \left[\tilde{x}_{i} \tilde{\gamma}_{i} / \tilde{x}_{i}^{(i)} \hat{\gamma}_{i}^{(i)}\right]-r_{i} \ln \left[\tilde{x}_{0} \tilde{\gamma}_{0} / \tilde{x}_{0}^{(i)} \tilde{\gamma}_{0}^{(i)}\right]
$$

где $x_{i}$ - молярная доля компонента $i$ в растворе, $\tilde{x}_{i}-$ то же, если дырки рассматриваются как сорт частиц.

Для выражения коэффициентов активности и энтальпий смешения используются те же соотношения, что и в решеточной модели без вакансий [6]

$$
\ln \tilde{\gamma}_{i}=\ln \tilde{\gamma}_{i \text { комб }}+\ln \tilde{\gamma}_{i \text { ост. }}
$$

Комбинаторный член, $\ln \tilde{\gamma}_{i}$ комб, описывается уравнением ГуггенгеймаСтавермана [?], а остаточный член выражается через

$$
\ln \tilde{\gamma}_{i \text { ост }}=z \sum_{s} q_{s i} \ln \left(\chi_{s} / \chi_{s i}\right)
$$

где $z$ - координационное число решетки, принятое здесь равным 10 , $q_{s i}$ - площадь поверхности группы $s$ в молекуле $i, \chi_{s}$ и $\chi_{s i}$ - решение системы квазихимических уравнений

$$
\chi_{s} \sum_{t=1}^{h} \alpha_{t} \chi_{t} \eta_{s t}=1
$$

для групп типа $s$ в растворе и в решетке, содержащей только частицы $i$ соответственно, $\alpha_{t}$ - поверхностная доля групп типа $t$ в растворе, $\eta_{s t}=$ $=\exp \left(w_{s t} / \kappa T\right)$.

Зависимость параметра $w_{s t}$ от температуры учитывается в форме

$$
\begin{gathered}
\frac{w_{s t}}{T}=\frac{w_{s t}^{0}}{T_{0}}+\frac{h_{s t}^{0}}{T_{0}} \frac{T_{0}-T}{T}+c_{s t}\left(\ln \frac{T_{0}}{T}-\frac{T_{0}-T}{T}\right), \\
h_{s t}=h_{s t}^{0}+c_{s t}\left(T-T_{0}\right),
\end{gathered}
$$

где $w_{s t}^{0}, h_{s t}^{0}, c_{s t}-$ энергетические параметры модели, называемые энергией, энтальпией и теплоемкостью взаимообмена, определенные при температуре $T_{0}$.

Энтальпия смешения рассчитывается через функции смешения, внутреннюю энергию $\Delta U^{M}$ и объем $\Delta V^{M}$ по уравнению

$$
H^{E}=\Delta U^{M}+P \Delta V^{M},
$$

$$
\begin{gathered}
\text { где } \Delta U^{M}=\sum_{s, t} \tilde{U}_{s t} \eta_{s t}\left(\tilde{A} \chi_{s} \chi_{t} \alpha_{s} \alpha_{t}-\sum_{i=1}^{h} \widetilde{A}^{(i)} \chi_{s}^{(i)} \chi_{t}^{(i)} \alpha_{s}^{(i)} \alpha_{t}^{(i)}\right) / 2, \\
\tilde{U}_{s t}=R\left[T_{0} h_{s t}+c_{s t}\left(T-T_{0}\right)\right] \\
\widetilde{A}^{(i)}=\left[\left(\widetilde{\nabla}^{(i)}-1\right) r_{i}+q_{i}\right] x_{i}, \quad \tilde{A}=(\widetilde{V}-1) \sum_{i=1}^{k} x_{i} r_{i}+\sum_{i=1}^{k} x_{i} q_{i},
\end{gathered}
$$

$q_{i}$ - площадь поверхности молекулы $i, \quad \widetilde{V}=V / v^{*} \sum_{i=1}^{k} x_{i} r_{i}-$ приведенный объем, $\Delta V^{M}=v^{*} \sum_{i=1}^{h}\left(\widetilde{\nabla}-\widetilde{\nabla}^{(i)}\right) r_{i} x_{i}$.

Молекулы 1-алкенов и 1-алкинов были разбиты на группы с кратными связями, $\mathrm{CH}_{2}=\mathrm{CH}, \mathrm{HC} \equiv \mathrm{C}$, и на группы « $\mathrm{CH}_{2}$ ». Последние включали в себя группы $\mathrm{CH}_{3}$ и $\mathrm{CH}_{2}$, которым приписывалась одинаковая энергетика взаимодействия с другими группами. За молекулами алканов сохранены 
ра́нее $\left.{ }^{3}\right]$ предложенная совокупность группі и установленные́ параметры взаимодействия. Температурное изменение энергетических параметров оценивалось с помощью выражений (8).

Геометрические параметры групп (табл. 1) $r_{s}$ определены по экспериментальным данным о давлении пара и плотности чистых компонентов $\left.{ }^{3}\right]$. Второй геометрический параметр, $q_{s}$, вычислен на основе соотношений

$$
\begin{gathered}
l_{s}=z\left(q_{s}-r_{s}\right) / 2-\left(r_{s}-1\right), \\
l_{i}=\sum_{s}\left(l_{s}-1\right) v_{s i}+1
\end{gathered}
$$

где $l_{i}$ - фактор объемности молекул $i$, равный нулю для линейных молекул, $l_{s}$ - фактор объемности групп $s, v_{s i}$ - число групп в молекуле $i$.

Таблица 1

\begin{tabular}{|c|c|c|c|}
\hline Группа & $r_{s}$ & $q_{s}$ & $l_{s}$ \\
\hline $\begin{array}{l}\mathrm{CH}_{3} \\
\mathrm{CH}_{2} \\
\mathrm{CH}_{2}=\mathrm{CH} \\
\mathrm{HC} \equiv \mathrm{C} \\
\text { дырка }\end{array}$ & $\begin{array}{l}1,9621 \\
1,0865 \\
2,7093 \\
2,2534 \\
1\end{array}$ & $\begin{array}{l}1,6697 \\
0,8692 \\
2,2674 \\
1,9027 \\
1\end{array}$ & $\begin{array}{l}0,5 \\
1,0 \\
0,5 \\
0,5 \\
0\end{array}$ \\
\hline
\end{tabular}

Геометрические параметры групп

Параметры взаимодействия групп (табл. 2) для смесей с 1-алкенами были определены по данным о температурной зависимости плотности $(\varrho)$ и давления пара $(P)$ 1-гексена, 1-октена и 1-децена в интервале температур 283-365 K [8] (параметры I), по данным об избыточной энергии Гиббса $\left(G_{328 \mathrm{~K}}^{E}\right)$ и энтальпии смешения $\left(H_{298 \mathrm{~K}}^{E}\right)$ трех составов раствора 1-гептен-н-гептан [9] (параметры II) и путем варьирования этих величин с данными о давлении пара 1-гептена при трех температурах (параметры III).

Параметры I воспроизводят исходные данные со следующей точностью:

$\begin{array}{ccc}\text { Алкен } & \delta \varrho, \% & \delta P, \% \\ \mathrm{C}_{6} & 0,3 & 0,1 \\ \mathrm{C}_{8} & 0,4 & 0,2 \\ \mathrm{C}_{10} & 0,8 & 0,6\end{array}$

С их помощью удалось вполне удовлетворительно описать концентрационные зависимости температур кипения и давления пара в ряде бинарных систем типа 1-алкен-н-алкан и 1-алкен-1-алкен (рис. 1, А-Д; рис. 2, А). Однако рассчитанные на их основе избыточные энергии Гиббса в системе 1-гептен-н-гептан лишь качественно согласуются с экспериментом (рис. 2, Б), а вычисленные энтальпии смешения имеют обратный знак. Параметры II хорошо воспроизвели концентрационную зависимость $G^{E}$ и $H^{E}$ в указанной системе (рис. 2, Б, В), но при этом увеличилось отклонение между расчетными и экспериментальными данными о зависимости $P=f\left(x_{i}\right)$ (рис. $\left.2, A\right)$. Удовлетворительные результаты при одновременном описании всех трех зависимостей получены с помощью параметров III (рис. 2, А-B).

Энергетические параметры групп с тройной связыю определены только по данным о чистых компонентах (табл. 2). Энергия взаимообмена $w_{s t}$ определялась по данным о плотности и давлении пара 1-гексина и 1-гептина при $T=333,15 \mathrm{~K}$. Затем по данным о температурной зависимости плотности и давления пара 1-гексина, 1-октина и 1-нонина в интервале 

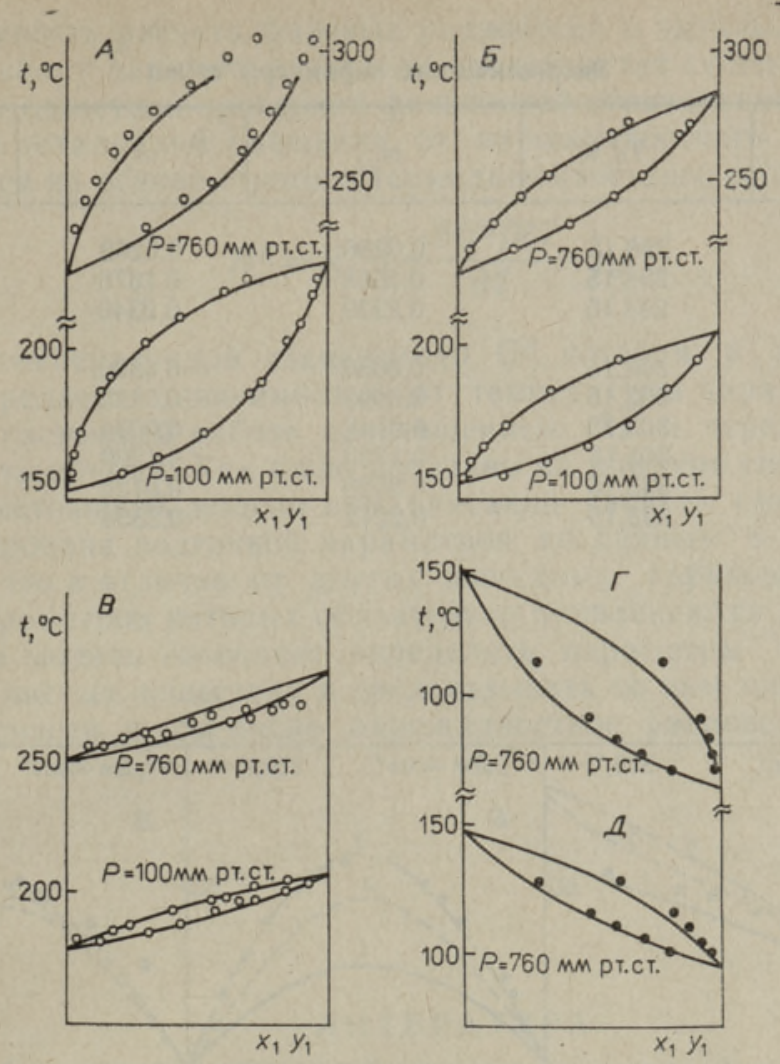

Рис. 1. Равновесие жидкость-пар в системах 1-октадецен-н-додекан (А), 1-гексадецен-н-додекан (Б), 1-гексадецен-н-тетрадекан (В), 1-гексен-1-нонен (Г), 1-гептен1 -нонен (Д). Экспериментальные данные: $\mathrm{O}\left[{ }^{10}\right]$, [11]. Кривая - расчет по дырочной модели. $x_{1}, y_{1}$ - молярная доля первого из названных компонентов бинарной системы в растворе и в паре (здесь и на рис. 2,3 ).

температур $293-344 \mathrm{~K}, 293-400 \mathrm{~K}$ и $293-423 \mathrm{~K}\left[{ }^{8}\right]$ соответственно были определены два других параметра взаимодействия - $h_{s t}$ и $c_{s t}$. Качество описания исходных данных характеризуют следующие средние относительные величины:

$\begin{array}{ccc}\text { Алкин } & \delta \varrho, \% & \delta P, \% \\ \mathrm{C}_{6} & 0,6 & 0,2 \\ \mathrm{C}_{8} & 0,2 & 0,1 \\ \mathrm{C}_{9} & 0,2 & 0,3\end{array}$

Әти параметры позволили вполне удовлетворительно предсказать концентрационную зависимость энтальпий смешения, коэффициентов активности компонентов, зависимость состава пара от состава раствора, а следовательно, и азеотропные составы в системах типа 1-алкин-н-алкан (рис. $3, \mathrm{~A}-\mathrm{E})$. Качественно верно передается характер температурной зависимости $G^{E}$ и $H^{E}$, хотя при большом перепаде температур расчет значительно превышает действительное изменение $H^{E}$, как видно на примере изменения $H^{E}$ эквимолярного раствора 1-гептин-н-гептан, Дж/моль:

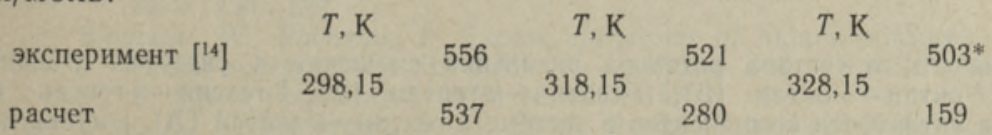

* Рассчитано из соотношения $H E=a+b T$, где $a, b$ - константы, определенные по экспериментальным данным об энтальпиях смешения [14]. 
Энергетические параметры групп

\begin{tabular}{|c|c|c|c|c|}
\hline Группы & $T_{0}, \mathrm{~K}$ & $w_{s t}$ & $h_{s t}$ & $c_{s t}$ \\
\hline $\begin{array}{c}\mathrm{CH}_{3} / \mathrm{CH}_{2} \\
\mathrm{CH}_{3} / \text { дырка } \\
\mathrm{CH}_{2} / \text { дырка } \\
\mathrm{CH}_{2}=\underset{\mathrm{CH} / \mathrm{CH}_{2}}{\text { (I) }} \\
\\
\text { (II) } \\
\text { (III) } \\
\mathrm{CH}_{2}=\mathrm{CH} / \text { дырка } \\
\mathrm{HC} \equiv \mathrm{C} / \mathrm{CH}_{2} \\
\mathrm{HC} \equiv \mathrm{C} / \text { дырка }\end{array}$ & $\begin{array}{l}298,15 \\
298,15 \\
298,15 \\
303,15 \\
303,15 \\
303,15 \\
303,15 \\
333,15 \\
333,15\end{array}$ & $\begin{array}{l}0,0056 \\
0,0195 \\
0,0142 \\
0,1730 \\
0,0520 \\
0,2212\end{array}$ & $\begin{array}{r}-0,0820 \\
0,0122 \\
0,0034 \\
0,1299 \\
0,0120 \\
0,2354\end{array}$ & $\begin{array}{r}0,0081^{*} \\
-0,0072^{*} \\
-0,0060^{*} \\
0,0268 \\
0,0268 \\
-0,6094 \\
-0,1064 \\
-1,2268 \\
-0,1363\end{array}$ \\
\hline
\end{tabular}

* Заимствованы из [5].

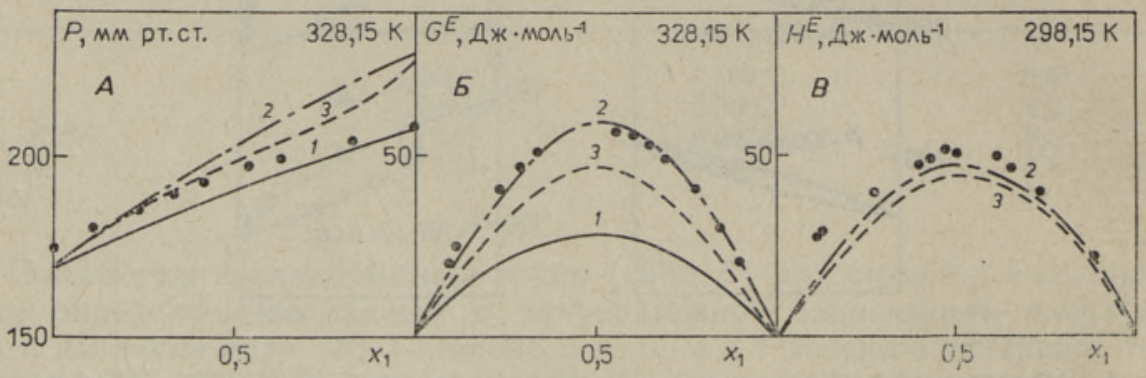

Рис. 2. Зависимость давления пара (А), избыточной энергии Гиббса (Б) и энтальпии смешения (В) от состава раствора 1-гептен-н-гептан.

Экспериментальные данные: [ ${ }^{9}$ ]. Расчет по дырочной модели с параметрами I (I), II (2) и III (3).

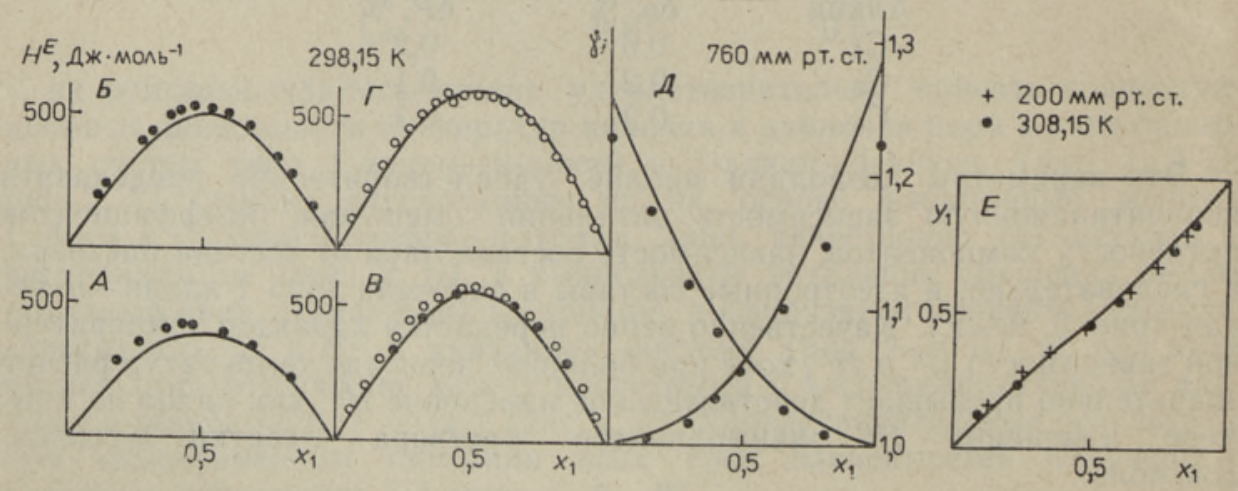

Рис. 3. Зависимость от состава раствора энтальпий смешения в системах 1-децинн-декан (А), 1-октин-н-октан (Б), 1-гептин-н-гептан (В), 1-гексин-н-гексан (Г), коэффициентов активности компонентов в системе 1-гептин-н-гептан (Д), состава пара в системе 1-гексин-н-гексан (Е). Экспериментальные данные: $\bigcirc\left[{ }^{12}\right], \times\left[{ }^{13}\right]$, $\left[{ }^{14}, 1^{5}\right]$. Кривая - расчет по дырочной модели. 
Возможность расчета фазовых равновесий и энтальпий смешения по единому набору параметров модели представляет существенный интерес и является известной проверкой физической обоснованности моделей $\left[{ }^{16}\right]$. Переход к энтальпиям смешения от парожидкостного равновесия осуществляется на основе строгого термодинамического соотношения

$$
H^{E}=R T^{2}\left(\frac{\partial G^{E} / R T}{\partial T}\right)_{P, x}
$$

с учетом температурной зависимости $G^{E}$, которая в рассматриваемой модели передается зависимостью от температуры параметра $w_{s t}$, выраженной в настоящей работе соотношением (8) и через температурную зависимость объема. Как было показано на примере системы 1-гептенн-гептан, неточность модели при описании свойств смесей может быть скомпенсирована подгонкой параметров по данным о смесях. Следует отметить, что в отличие от других известных групповых моделей, для оценки параметров которых обязательно привлекаются данные о смесях, настоящая модель позволяет определять параметры также только по данным о чистых веществах и предсказывать по ним термодинамические свойства смесей, в том числе парожидкостное равновесие и энтальпии смешения.

\section{Л И ТЕ Р А Т Р А}

1. Derr, E. L., Deal, C. H. Analytical solutions of groups. Correlation of activity coefficients through structural group parameters // Proc. Int. Symp. Distill., 1969, 3, 40-51 (Inst. ENG. London).

2. Fredenslund, Aa., Jones, R. L., Prausnitz, J. M. Group contribution estimation of activity coefficients in nonideal liquid mixtures // AIChE Journal, 1975, 21, N 6, 1086-1099.

3. Smirnova, N. A., Victorov, A. I. Thermodynamic properties of pure liquids and solutions from the hole group-contribution model // Fluid Phase Equil., 1987, 34, $235-263$.

4. Смирнова Н. А., Викторов А. И. Расчеты термодинамических свойств жидкостей и растворов на основании дырочной квазихимической модели. 2. н-Аліканы // Ж. физ. хим., 1986, 60, № 5, 1096-1099.

5. Смирнова Н. А., Викторов А. И. Расчеты термодинамических свойств жидкостей и растворов на основании дырочной квазихимнческой модели. 3. Смеси н-алканов // Ж. физ. хим., 1986, 60, 1100-1102.

6. Кудрявцева Л., Куус М., Пиотровская Е., Кирсс Х. Расчет термодинамических свойств систем, содержащих алкены, с помощью групповых моделей раствора. 2. Квазихимическая групповая модель // Изв. АН ЭССР. Хим., 1987, 36, № 4, $241-249$.

7. Staverman, A. J. The entropy of high polymer solutions. Generalisations of formulae// Rec. Trav. Chim. Pays-Bas, 1950, 69, N 2, 163-174.

8. Основные физико-химические свойства индивидуальных изомеров нормальных алкенов и алкинов, алкилцикленов, алканолов и алкенолов. Таллин, 1986.

9. Kudrjavtseva, L., Kuus, M., Viit, H., Eisen, O. 1-Heptene + heptane. Liquid-vapor equilibrium, excess Gibbs energy and excess enthalpy // Int. Data Series. Ser. A. Selected Data on Mixtures, 1981, 1980, N 1, 13-16.

10. Коган В. Б., Фридман В. М., Кафаров В. В. Равновесие между жидкостью и паром. M.-Л., 1966.

11. Кудрявцева Л., Куус М., Эльвельт А. О межмолекулярных взаимодействиях в системах, содержащих нормальные алкены // Изв. АН ЭССР. Хим., 1983, 32, № 4, 259-267.

12. Woycicki, W., Rhensius, P. Excess enthalpies of binary mixtures containing unsaturated hydrocarbons. 3. n-Alkane- $n$-alkyne and tetrachloromethane- $n$-alkyne // J. Chem. Thermodyn., 1979, 11, 153-158.

13. Otsa, E., Kudrjawzewa, L. S., Eisen, O. G., Piotrowskaia, E. M. Thermodynamische Untersuchungen an $n$-Alkan/n-Alkin-Systemen. 2. Isobare Dampf-Flüssigkeitsgleichgewichte // Monatsh. Chem., 1980, 111, N 3, 607-617. 
14. Otsa, E., Kudrjawzewa, L., Eisen, O. Thermodynamische Untersuchungen an n-Alkan/n-Alkin-Systemen. 1. Die Mischungswärmen // Monatsh. Chem., 1980, 111, $\mathrm{N} 1,37-42$.

15. Кудрявцева Л., Вийт Х., Эйзен О. Равновесие жидкость-пар в бинарных системах, образующихся при синтезе $\alpha$-алкенов // Изв. АН ЭССР. Хим. Геол., 1968, 17, № 3, 242-250.

16. Nicolaides, G. L., Eckert, C. A. Optimal representation of binary liquid mixture nonidealities // Ind. Éng. Chem., 1978, 17, N 4, 331-340.

Ленинградский государственный университет

Поступила в редакцию

Институт химии

Академии наук Эстонской ССР

$12 / \mathrm{I} 1989$

A. VIKTOROV, L. KUDRJAVTSEVA, M. KUUS

\section{VAKANTSIDEGA KVAASIKEEMILISE GRUPIMUDELI RAKENDAMINE KOLLASTUMATUID SUSIVESINIKKE SISALDAVATELE SOSTEEMIDELE}

Auru ja vedeliku tasakaalu (AVT) ning segunemisentalpia $(H E)$ arvutamiseks 1-alkeenide ja 1-alküünide segudes $n$-alkaanidega on kasutatud vakantsidega kvaasikeemilist grupimudelit. On määratud mudeli energeetilised parameetrid gruppidele $\mathrm{CH}_{2}=\mathrm{CH}$ ja $\mathrm{HC} \equiv \mathrm{C}$. Kasutades parameetreid, mis on arvutatud ainult puhaste vedelike omaduste alusel, on saadud rahuldav AVT kirjeldus 1-alkeene sisaldavates süsteemides. Nende parameetrite abil on segudes 1 -alküün- $n$-alkaan ōnnestunud ennustada samaaegselt nii AVT kui ka $H^{E}$ väärtusi.

\section{A. VICTOROV, L. KUDRYAVTSEVA, M. KUUS}

\section{APPLICATION OF HOLE GROUP-CONTRIBUTION MODEL TO THE SYSTEMS WITH UNSATURATED HYDROCARBONS}

To calculate vapor-liquid equilibria (VLE) and enthalpies of mixing $(H E)$ in the systems of 1 -alkenes and 1 -alkynes with $n$-alkanes the hole group-contribution model has been used. The $\mathrm{CH}_{2}=\mathrm{CH}$ and $\mathrm{HC} \equiv \mathrm{C}$ group energetic parameters have been determined. The parameters, estimated from pure liquid properties only, give satisfactory results of VLE calculations for the systems with 1-alkene, and enable sufficient agreement with experiment for the simultaneous prediction of VLE and $H^{E}$ in 1-alkyne-n-alkane mixtures. 